\title{
Identification of infectious Pseudomonas aeruginosa strains in an occupational saturation diving environment
}

\author{
Catrine Ahlén, Lise Helen Mandal, Ole Jan Iversen
}

\begin{abstract}
Objectives-Occupational saturation divers have various skin disorders, of which skin infections are the most serious and frequent. Pseudomonas aeruginosa is the microbe most often isolated from skin infections in divers. The purpose of the present work was $(a)$ to report the occurrence of $P$ aeruginosa in skin infections in operational saturation diving in the North Sea from 1987 to 1995; (b) to report the environmental occurrence of $P$ aeruginosa in saturation diving systems, and finally (c) to identify possible relations between infection related to strains of $P$ aeruginosa and environmental isolates of the microbe.
\end{abstract}

Results-During the period 1987-95, $P$ aeruginosa was isolated from 257 skin infections in operational saturation divers. Most of the isolates related to infection by $P$ aeruginosa show a unique growth inhibition pattern towards the normal skin flora, and the serotype pattern of $P$ aeruginosa from skin infections is limited compared with similar infections in non-divers. In a miniepidemiological study on board one diving vessel during one operational diving period, five significantly different DNA fragment profiles were found among the 12 isolates related to infection by $P$ aeruginosa obtained from the saturation system. In two cases the infectious genotypes were detected in the fresh water for the saturation chambers weeks before the arrival of the infected diver.

SINTEF Unimed, Extreme Work Environment, N-7034 Trondheim, Norway C Ahlén

L H Mandal

Norwegian University of Science and Technology, Department of Microbiology, N-7006 Trondheim, Norway O J Iversen

Correspondence to: Dr Catrine Ahlén, SINTEF Unimed, Extreme Work Environment, N-7034 Trondheim, Norway.

Accepted 12 January 1998 in saturation environments. PFGE
analyses were shown to be superior both to antibacterial factor and to serotyping in epidemiological analyses of $P$ aeruginosa infections in saturation diving.

(Occup Environ Med 1998;55:480-484)

Keywords: Pseudomonas aeruginosa; skin infections; saturation diving; pulsed field gel electrophoresis; serotyping
Saturation diving is extensively used for maintenance and inspection of North Sea off shore sub-sea oil production systems. The saturation period includes compression, bottom time (working period), and decompression. A typical saturation period is between 12 and 24 days, during which the divers live in pressurised chambers on board a diving vessel in an atmosphere of pressurised helium and oxygen. ${ }^{1}$ When working, the divers dress in a protective diving suit, in which heated $\left(\sim 36^{\circ} \mathrm{C}\right)$ sea water is continuously flushed on to the divers' skin to maintain thermal balance. The ambient absolute pressure $(0.6-2.1 \mathrm{Mpa})$ is dependent on the working depth, which normally in the North Sea is $50-200 \mathrm{~m}$. The partial pressure of oxygen will vary between 20 and 50 pa depending on the phase of the dive. The ambient temperature in the chambers varies with the working depth, but will often be around $28-30^{\circ} \mathrm{C}$. The relative humidity is generally high and may reach $80 \%-90 \%$, but is normally set to be $<50 \%$.

Occupational saturation divers working in the Norwegian part of the North Sea report several health complications, of which skin infections and muscle pains are reported most often. ${ }^{2}$ In the same population, long term effects on the nervous system and pulmonary function from this occupational exposure have been reported, ${ }^{3}$ as well as influence on the immune system. ${ }^{4}$ We have recently described a unique skin syndrome (Diver's hand) among the occupational saturation divers. ${ }^{5}$

A specific skin infection-namely, external otitis - is well known from deep diving. ${ }^{6}{ }^{7}$ Other superficial skin infections of the face and neck are less well known. ${ }^{8}$ Since 1987 , we have performed a regular control programme for infection, disinfection, and hygiene among several of the diving contractors operating on the Norwegian offshore shelf in the North Sea. Our objectives are to describe the occurrence of Pseudomonas aeruginosa infections in occupational saturation diving and to present new knowledge about how strains related to infections are introduced into the saturation systems. insufficient for epidemiological purposes
Material and methods

DIVING ACTIVITY

From Norwegian Petroleum Directorate statistics, ${ }^{2}$ a total number of 1340000 man hours saturation diving have been registered on the Norwegian offshore shelf during the period 1987-95. The diving activity has involved several diving contractors, each using two or more 
diving vessels. A mean saturation period of 12 days, with a mean crew of six divers gives 775 saturation periods within the given period.

MICROBIOLOGICAL INVESTIGATIONS

Sampling

The microbial material from the samples included skin infections, which presented as external otitis as well as folliculitis and abcesses mostly localised to the face and neck areas. Also, regular environmental control samples of fresh water, chamber interior, chamber atmosphere, and divers' personal equipment-for example, helmets, masks, and hat liners - were included.

Microbial sampling was performed by a licensed medic on board the diving vessels. The samples from infections and the environment were taken with culturettes (BectonDickinson) and transported by express (within $24 \mathrm{~h}$ ) to the clinical microbiological laboratory onshore (Department of Microbiology, University Hospital, Trondheim). Cultivation and typing were done with conventional microbiological and biochemical methods.

\section{Serotyping}

Pseudomonas aeruginosa was serotyped with commercial antisera against $\mathrm{O}$-antigens 1-17 (Difco Laboratories, Detroit, MI). The serotyping was performed according to the method described by the manufacturer.

Bacterial growth inhibition test

Interference between $P$ aeruginosa and normal skin flora was investigated routinely. The test was performed as a competitive well plate assay with coryneforms or staphylococcal strains (C: NDCF, C: NDCR, or S:NDSF) as indicator strains. The test was prepared by flooding or swabbing a 4 hour inoculum broth with the indicator strain over the surface of a blood agar plate. The plates were then allowed to dry in air. Pseudomonas aeruginosa broth cultures were added in $25 \mu \mathrm{l}$ wells. Pseudomonas aeruginosa ATCC 27853 was used as a negative control. The plates were incubated at $37^{\circ} \mathrm{C}$ overnight, and zones of inhibition of coryneforms or staphylococcal growth were recorded.

\section{Genotyping of $P$ aeruginosa by pulsed field gel electrophoresis (PFGE)}

The method was performed essentially as described by Kaufmann and Pitt $1994^{9}$ with minor modifications.

Pseudomonas aeruginosa DNA preparationDNA was prepared from overnight cultures on solid medium (blood agar). The final bacterial concentration was set to $\sim 1 \times 10^{9} \mathrm{cfu} / \mathrm{ml}$ in SE buffer (75 mM NaCl, $25 \mathrm{mM}$ EDTA ( $\mathrm{pH}$ 7.4)). The bacterial suspension was then mixed with $2 \%$ (wt/vol) low melting point agarose, and applied into plug moulds and solidified. Lysis of the bacteria was performed with proteinase $\mathrm{K}$ (Sigma) in a modified EC lysis buffer at $56^{\circ} \mathrm{C}$ overnight. The DNA plugs were then washed four times in TE buffer $(10 \mathrm{mM}$ Tris, $1 \mathrm{mM}$ EDTA (pH 7.5)), each for 30 minutes at room temperature. The DNA plugs were then stored in TE buffer at $4^{\circ} \mathrm{C}$ until restriction endonuclease analyses.

Digestion of DNA with restriction endonuclease was mainly done with the rare-cutter endonuclease SpeI (Boehringer Mannheim Biochemica). For verification of the results, another five endonucleases: AsnI, DraI, XbaI, SspI, and DpnI (Boehringer Mannheim Biochemica) were used. The restriction enzyme treatment was done according to the procedure recommended by the producer. The DNA plugs were cut and digestion was performed in $100 \mu \mathrm{l}$ restriction enzyme buffer with $10 \mathrm{U}$ SpeI, in a $37^{\circ} \mathrm{C}$ water bath for 4 hours.

Gel electrophoresis - The separation of DNA fragments was performed in $1.2 \%$ agarose in a modified Tris-borate-EDTA (TBE) buffer $(0.25 \times)$ in a commercial BIORAD CHEF DR TA. ${ }^{10}$ A linear ramping from $5-25 \mathrm{~s}$ for 20 hours run time followed by $5-60 \mathrm{~s}$ for 17 hours, at a voltage of $5.3 \mathrm{~V} / \mathrm{cm}$ and a running temperature of $14^{\circ} \mathrm{C}$. A $\lambda$ ladder (PFG Marker, Biolabs) was applied to each gel as standard, and a reference strain of $P$ aeruginosa (ATCC 27853) was initially analysed in parallel.

The gels were stained in ethidium bromide according to the original description. ${ }^{10}$ Destaining was done in distilled water for 2 hours. The gel was photographed under UV transillumination. Comparison of DNA fragment patterns was done by visual comparison of the PFGE patterns.

\section{Results}

ISOLATION OF PSEUDOMONAS AERUGINOSA IN SATURATION DIVING

During the period 1987-95, P aeruginosa was identified in 257 skin infections from operational saturation divers. More than $80 \%$ of the samples were pure isolates. Furthermore, $P$

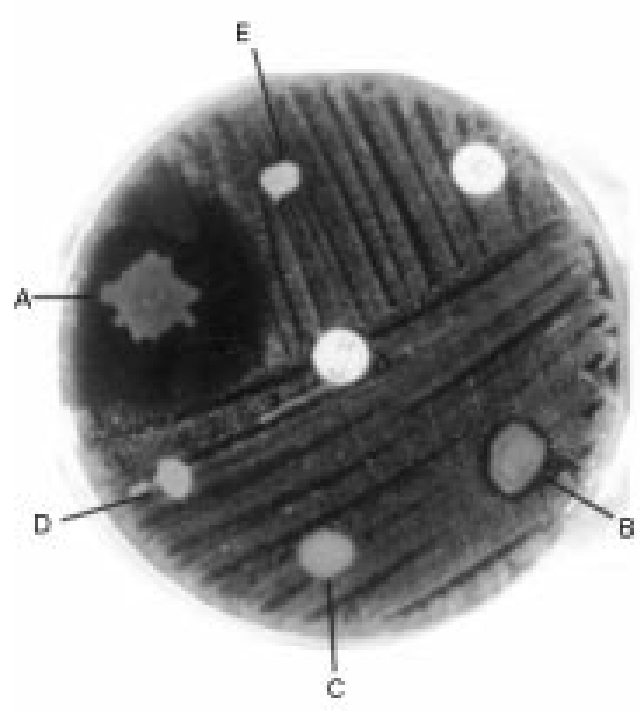

Figure 1 Detection of antibacterial factor $(A B F)$ from Pseudomonas aeruginosa isolates from divers' ear infection. Indicator strain S:NDSF (A) Isolate from divers skin infection to (B) $P$ aeruginosa ATCC 27853, (C) $P$ cepacia, and $(D, E)$ coagulase negative staphylococci from divers normal skin flora. 
A
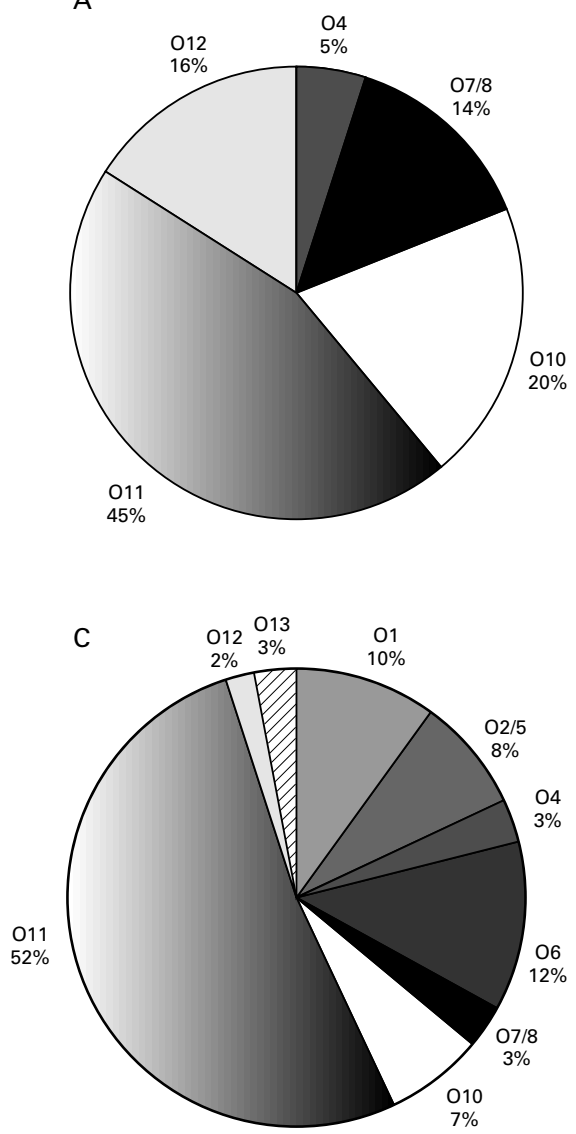

B
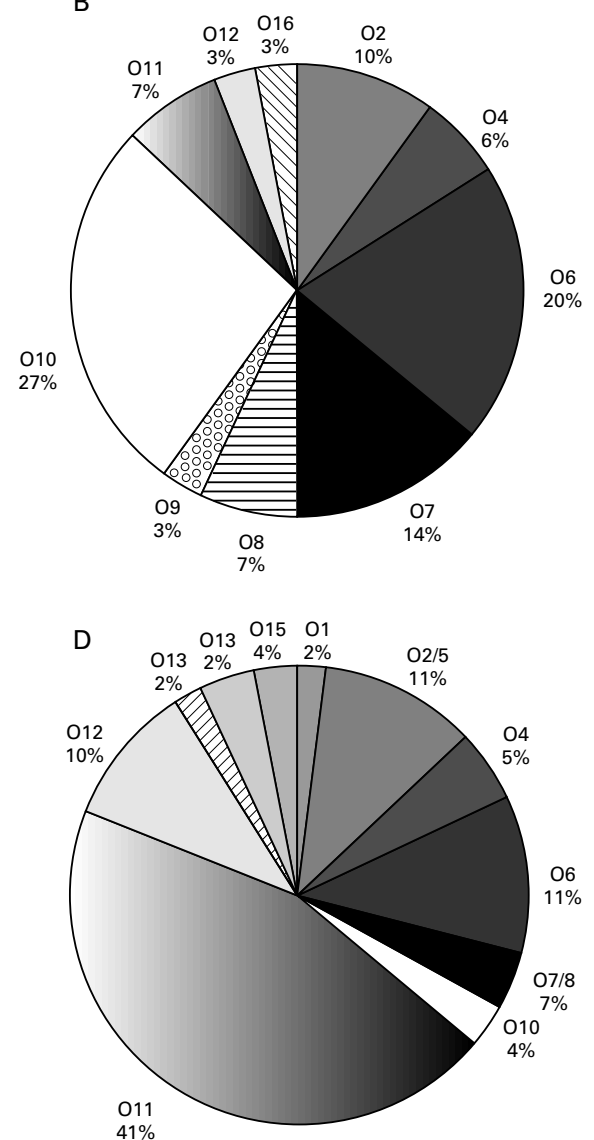

$41 \%$

Figure 2 Pseudomonas aeruginosa O serotypes from $(A)$ divers'skin infections $(n=120)$, (B) from skin infections in non-divers $(n=45),(C)$ from fresh water into chambers $(n=86)$, and $(D)$ from the occupational saturation environment $(n=150)$.

aeruginosa was present in more than $85 \%$ of the total samples from treated skin infections in divers (data not shown).

Analyses of fresh water and chamber environments throughout the period showed many various micro-organisms that were always present (data not shown). In this paper, attention has been paid only to $P$ aeruginosa, which was detected in an average of $35 \%-40 \%$ of the environmental samples with more from the earlier years of the survey (1987-9) and less during the following years, due to optimised cleaning, disinfection, and hygiene procedures. $^{10}$

\section{Antibacterial factor(s)}

Most of the $P$ aeruginosa isolates related to infection showed a unique growth inhibition pattern towards the normal skin flora-for example, coryneforms and coagulase negative staphylococci (fig 1).

This antibacterial factor (ABF) could be found in all skin infections in divers from whom $P$ aeruginosa was the only infection isolate, whereas it was only rarely found in comparable infections among non-divers (clinical or hospital material). The ABF was seldom found in $P$ aeruginosa isolates obtained from patients in hospital $(<20 \%)$ and the $P$ aeruginosa isolates from skin infections with mixed bacterial flora, as well as the $P$ aeruginosa environmental strains from saturation diving systems.

SEROTYPING OF P AERUGINOSA

From the divers' skin infections, a few $P$ aeruginosa serotypes are represented (fig $2 \mathrm{~A}$ ). Serotype O11 is abundant, but also serotypes $\mathrm{O} 10, \mathrm{O} 12, \mathrm{O} 4$ and $\mathrm{O} 7 / 8$ have been found with varying frequency. Compared with the isolates from clinical infections among non-divers, the serotypes present in saturation diving show a significantly limited pattern (fig $2 \mathrm{~B}$ ). From the saturation environment, serotype $\mathrm{O} 11$ is again abundant, but here several additional serotypes are represented, among which serotype $\mathrm{O6}$ is often found (fig $2 \mathrm{C}$ and D). Minor variations were found in the occurrence of serotypes from one vessel to another.

GENETIC MAPPING OF ISOLATES OF PSEUDOMONAS AERUGINOSA

Genetic mapping was performed of the strains of $P$ aeruginosa isolated from the saturation diving system on board one diving vessel stationed on the North Sea offshore sector, during consecutive operational diving periods in February to July 1994 (fig 3). During this period, 14 skin infections were reported and analysed. Pseudomonas aeruginosa was isolated from 12 of these infections. During the same period, $P$ aeruginosa was isolated from nine of 
A

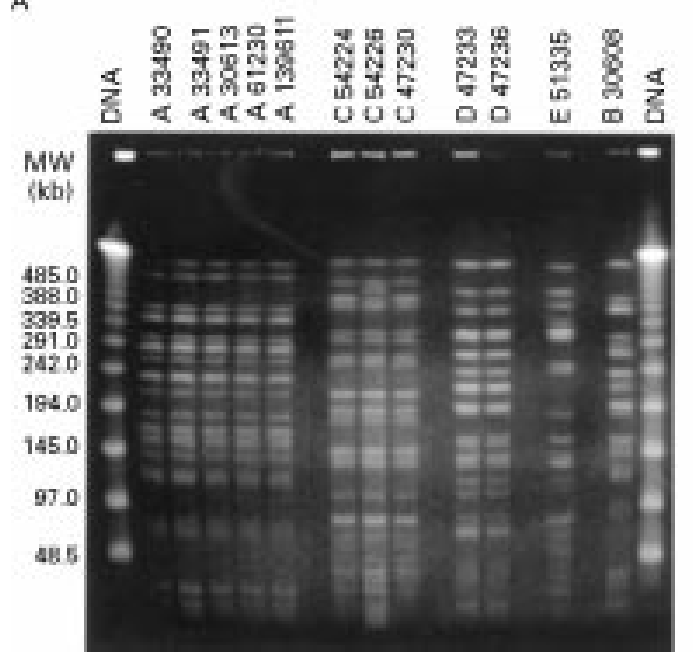

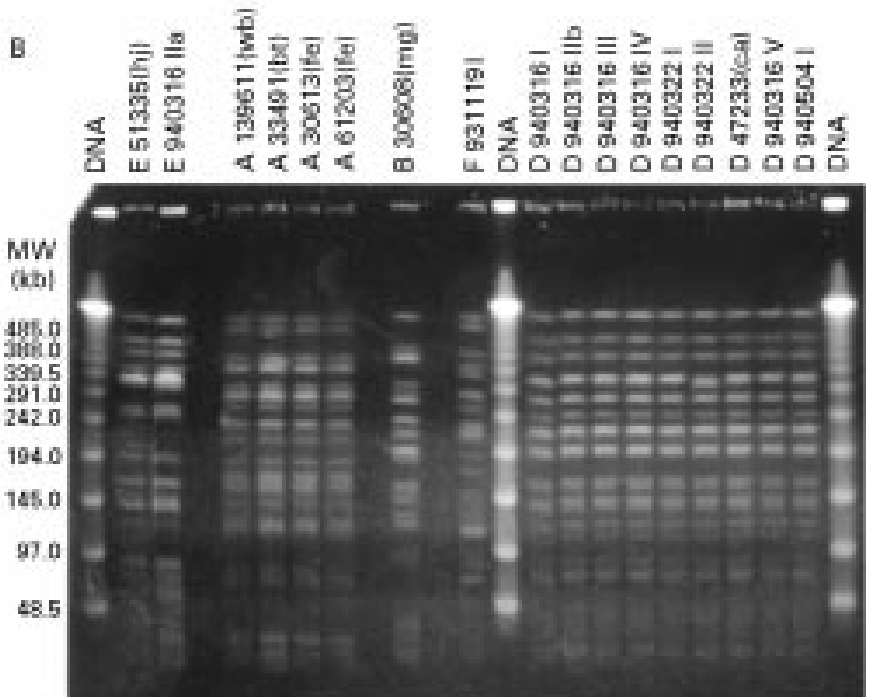

Figure 3 (A) Genetic mapping of Pseudomonas aeruginosa isolates from divers skin infections (PFGE patterns A-E), (B) and genetic mapping of $P$ aeruginosa from saturation environment and infections.

DNA fragment profiles among Pseudomonas aeruginosa strains related to infection

\begin{tabular}{|c|c|c|c|c|c|}
\hline Date & $A^{*}$ & $B$ & C & $D$ & $E$ \\
\hline 2 March 94 & $\mathrm{i}$ & & & & \\
\hline 3 March 94 & & $\mathrm{i}$ & & & \\
\hline \multirow[t]{2}{*}{6 March 94} & $\mathrm{i}$ & & & & \\
\hline & $\mathrm{i}$ & & & & \\
\hline 16 March 94 & & & & $\mathrm{w}$ & $\mathrm{w}$ \\
\hline 22 March 94 & & & & $\mathrm{w}$ & \\
\hline 12 April 94 & & & & $\mathrm{i}$ & \\
\hline 13 April 94 & & & $\mathrm{i}$ & $\mathrm{i}$ & \\
\hline 19 April 94 & & & & & $\mathrm{i}$ \\
\hline \multirow{2}{*}{28 April 94} & & & $\mathrm{i}$ & & \\
\hline & & & $\mathrm{i}$ & & \\
\hline 4 May 94 & & & & e & \\
\hline 14 May 94 & $\mathrm{i}$ & & & & \\
\hline 25 July 94 & & & & $\mathrm{i}$ & \\
\hline
\end{tabular}

${ }^{\star}$ Genotyping of Pseudomonas aeruginosa isolates (A-E) obtained from skin infections (i), fresh water (w), and the environment (e).

110 environmental samples (fresh water and environment).

Five different DNA fragment profiles were found among the 12 analysed isolates related to infection by $P$ aeruginosa. The profiles were verified by use of several endonucleases. The profiles were consecutively coded from A to E, starting with $\mathrm{A}$ for the first infection within the period of investigation. Figure 3 A shows PFGE analysis of the infection isolates when grouped by their DNA fragment profiles. Two of the patterns related to infection were also found in the environmental strains; eight of the nine $P$ aeruginos a environmental strains were of the same PFGE pattern (D), whereas one strain was shown to be pattern E (fig $3 \mathrm{~B}$ ). The D pattern strains were spread throughout all the saturation chamber system, resulting in several isolates from the same sampling date (table and fig $3 \mathrm{~B}$ ).

Table 1 shows that some infectious strains were detected in the fresh water several weeks before the infections and even before the arrival of the infected divers at the vessel.

\section{Discussion}

In cooperation with saturation diving companies operating offshore on the North Sea, a long term continuous surveillance of skin infections in occupational saturation divers has been carried out. During a nine year period, microbes from skin infections and regular microbiological samples from the saturation system environment have been analysed. More than 250 skin infections have been analysed during this period.

The dominant microbe in divers' skin infections is Paeruginosa, and in this study, attention has been paid only to this microbe. Pseudomonas aeruginosa is seldom found in skin infections in general, and it is not easy to experimentally induce infection by this microbe in otherwise healthy skin. ${ }^{112}$ The microbial flora within saturation systems is very rich, both in species and numbers, and $P$ aeruginosa is only a minor part. The dominance of this microbe in infections can therefore hardly be explained simply from microbial load. Increase in microbial growth rate of $P$ aeruginosa is experimentally shown in pressurised heliox compared with normobaric air (unpublished data). The characteristic growth inhibiting feature, antibacterial factor, which is common among infection strains from divers, although much less frequent in environmental isolates in saturation systems, strongly indicates that qualitative rather than quantitative properties are of importance for infections. The fact that antibacterial factor could be found irrespectively of biotype, serotype, and pyocintype (unpublished observation), made it a useful marker in the infection control programme in the saturation diving systems.

Serotyping, which for almost a century has been a classic tool for epidemiological studies of $P$ aeruginosa, showed that only certain serotypes of $P$ aeruginos a are represented in the skin infections in saturation divers compared with similar infections in non-divers. Serotype O11 is dominant in skin infections in our material. The same serotype is also dominant in the occupational saturation environment. Increased frequency of certain serotypes in saturation environments may indicate that $\mathrm{O}$ 
antigens are of significance for the survival of the strains. Serotype O11 has also been reported as the single most common $P$ aeruginosa serotype in outbreaks of skin infection (folliculitis and ecthyma gangrenosum) in swimming pools and whirlpools world wide. ${ }^{14} 15$

The 12 strains related to infection investigated by genotyping could be grouped into five different patterns: A; B; C; D; and E. Some of these strains were shown to cause infections in several divers. Some of the strains were found during longer periods, and one strain was registered for more than four months within the same saturation chamber system. In two cases, the infection strains were isolated from the fresh water in the chambers more than one month before the infections. This shows that infection strains can persist in the fresh water system for several saturation periods and thereby constitute a persistent infection challenge through the divers' personal hygiene (daily showering).

To summarise, the findings described in this paper are of great importance for understanding how the infectious strains are introduced into the occupational saturation environment. Serotyping gives limited knowledge of distribution of the microbes in the saturation system as shown by the fact that the three genotypes A,B, and $\mathrm{E}$ all were serotype $\mathrm{O} 11$. Based on the data presented in this study, it is our opinion that genotyping is a necessity for epidemiological studies of $P$ aeruginosa in occupational saturation environments. To our knowledge, this is the first evidence that infectious strains are not necessarily introduced into the saturation environment by an infected diver.
We thank Statoil, Norsk Hydro, Saga Petroleum, and the Norwegian Petroleum Directorate for financial support. We also thank all the participating divers and the health personnel on board the diving vessels for their most skilful cooperation.

1 Freitag M, Woods A. Commercial diving. Reference and operations handbook. Bath, UK: Wiley, 1983.

2 Norwegian Petroleum Directorate. Report from the Dive data base-DSYS. Stavanger, Norway: Graphic center, NPD, 1995.

3 Hope A, Lund T, Elliott D, et al. Long term health effects of diving. An international consensus conference, Godøysund, Norway, 1993 fune 6-10. Bergen, Norway: NUTEC and University of Bergen, 1993.

4 Benestad HB, Hersleth IB, Hardersen H, et al. Functional capacity of neutrophil granulocytes in deep-sea divers. Scand F Clin Invest 1990;50:9-18.

5 Ahlén C, Iversen OJ, Risberg J, et al. Diver's hand: a skin disorder common in occupational saturation diving. Occup Environ Med 1998;55:141-3.

6 Thalman ED. A prophylactic program for the prevention of otitis externa in saturation divers. Washington, DC: US Navy Experimental Diving Unit, Washington Navy Yard, 1974. (Research report 10-74.)

7 Alcock SR. Acute otitis externa in divers in the North Sea. A microbiological survey of seven saturation dives. Fournal of Hygiene (Camb) 1977;78:395-409.

8 Ahlén C. Programme for research and development in diving technology (FUDT) bacteriology, 1988-90. A summary. 1991. (SINTEF report no STF23, A91039, ISBN no 82-5957099-8.)

9 Kaufmann ME, Pitt TL. Pulsed field gel electrophoresis of bacterial DNA. In: Methods in practical laboratory bacteriology. Boca Raton: CRC Press, 1994.

10 Ahlén C, Leinan I, Berg M. Guidelines for cleaning/disinfection in chamber systems used in operational saturation diving. Richardson, TX: Society of Petroleum Engineers, 1991. (SPE 23234.)

11 Singh G. Pseudomonas infection of skin: an experimental study. Int f Dermatol 1974;13:90-3.

12 Hojyo-Tomoka MT, Marples RR, Kligman AM. Pseudomonas infection in superhydrated skin. Arch Dermatol 1973;107:723-7.

13 Ratnam S, Hogan K, March SB, et al. Whirlpool-associated folliculitis caused by Pseudomonas aeruginosa: report of an outbreak and review. F Clin Microbiol 1986;23:655-9.

\section{Rejected manuscripts}

From February 1994, authors whose submitted articles are rejected will be advised of the decision and one copy of the article, together with any reviewer's comments, will be returned to them. The fournal will destroy remaining copies of the article but correspondence and reviewers' comments will be kept. 